\title{
O cuidado no domicílio: a visão da pessoa dependente e do cuidador
}

\section{The domicile care: the vision of the dependent person and the caregiver}

\section{Cuidado en el domicilio: la visión del dependiente y del cuidador}

\author{
Silvia Helena Zem-Mascarenhas', Ana Claudia T. Barros"
}

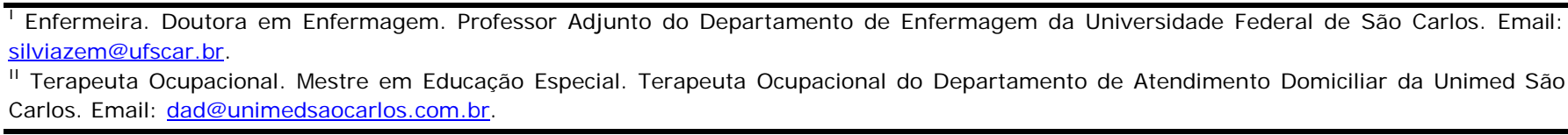

\section{RESUMO}

Assistência domiciliar caracteriza-se por um conjunto de procedimentos possíveis de serem realizados no domicílio, por equipe multiprofissional. Estudo de caráter descritivo exploratório, realizado no período de abril a agosto de 2006, com objetivo de caracterizar a pessoa dependente em atendimento domiciliar e seu respectivo cuidador, conhecer as percepções dessa população quanto à qualidade do serviço prestado na assistência domiciliar e conhecer como o cliente avalia o cuidado prestado por seu cuidador. Participaram do estudo 40 dependentes matriculados em um serviço de atendimento domiciliar do interior do estado de São Paulo e seus cuidadores. Os resultados revelaram que a maioria dos participantes pertencia à faixa etária de 61 a 70 anos, eram viúvos e apresentavam patologias variadas. $\mathrm{O}$ atendimento domiciliar foi considerado satisfatório na visão das pessoas dependentes e cuidadores e os conteúdos dos relatos foram agrupados em três categorias: atenção e cuidado da equipe, conforto do lar e "desospitalização". A assistência prestada pelo cuidador foi considerada ótima segundo a visão da pessoa dependente. Este estudo reflete a preocupação constante da equipe do atendimento domiciliar em proporcionar a melhora contínua da qualidade do serviço e satisfação das pessoas dependentes e seus cuidadores.

Descritores: Assistência domiciliar; Cuidadores; Serviços de assistência domiciliar.

\section{ABSTRACT}

Home care is characterized by a group of procedures that can be developed at home, by multiprofessional team. This descriptive exploring study was conducted between April and August of 2006, that aimed to characterize the customer in home nursing and his respective caregiver, get to know this population perceptions concerning the quality of the given home care services and how the customer evaluates the care offered by his caregiver. Forty customers registered in home nursing service from the countryside of São Paulo and their caregivers took part in the study. The results revealed that most customers were aged from 61 to 70 years old, were widowers and presented diverse pathologies. The home nursing was considered satisfactory according to the customers and their caregivers, and the content of their stories were grouped into three categories: team attention and care, home comfort and dishospitalization. The assistance given by the caregiver was considered satisfactory according to the clients' point of view. They have also emphasized that the customer-caregiver relationship is considered terrific as well. This study reflects a constant concern from the home nursing team willing to promote the continuous improvement of the service quality, and the dependants and their caregivers' satisfaction.

Descriptors: Home nursing; Caregivers; Home care services.

\section{RESUMEN}

La atención domiciliar es caracterizada como un conjunto de procedimientos que pueden ser realizados en la casa, por un equipo multiprofesional. Este estudio, de carácter exploratorio descriptivo, desarrollado en el periodo de abril a agosto de 2006, tuvo como objetivo caracterizar el dependiente en atención domiciliar y su respectivo cuidador; conocer las percepciones de ese grupo en relación a la calidad del servicio prestado en la asistencia domiciliar y conocer como los dependientes evalúan el cuidado prestado por el cuidador. Participaron del estudio 40 dependientes inscriptos en un servicio de atención domiciliar del interior del estado de São Paulo y sus cuidadores. Los resultados revelaron que la mayoría de los dependientes tenían entre 61 y 70 años, eran viudos y presentaban patologías variadas. La atención domiciliar fue considerada satisfactoria por los dependientes y cuidadores, y los contenidos fueron agrupados en tres categorías: atención y cuidados del equipo, confort del hogar y deshospitalización. La asistencia ofrecida por el cuidador fue considerada óptima según la visión del dependiente. Este estudio refleja una preocupación constante del equipo de la atención domiciliaria con el propósito de proporcionar la mejora continua de la calidad del servicio y la satisfacción de los dependientes y sus cuidadores.

Descriptores: Atención domiciliaria de salud; Cuidadores; Servicios de atención de salud a domicilio. 


\section{NTRODUÇÃO}

Assistência domiciliar consiste em prover a atenção à saúde de pessoas doentes, de alguma forma incapacitadas ou com doenças crônicas de qualquer idade, que sejam dependentes de assistência em seu domicílio, proporcionando condições para tratamento efetivo(1).

Segundo a Agência Nacional de Vigilância Sanitária(2), a assistência domiciliar caracteriza-se por um conjunto de atividades de caráter ambulatorial, programadas e continuadas desenvolvidas em domicílio.

A assistência domiciliar é realizada com vistas à promoção, manutenção e/ou restauração da saúde da pessoa dependente, com o objetivo de favorecer sua independência e preservar sua autonomia(3).

Trata-se de uma assistência que surgiu em resposta à individualização da assistência prestada; aliada à possibilidade da pessoa dependente/família manter maior controle sobre o processo de tomada de decisões relacionadas ao cuidado; à conseqüente diminuição dos custos quando comparados domicílio e hospital; redução dos riscos de infecção hospitalar; bem como ao maior envolvimento da família no planejamento e execução dos cuidados necessários ${ }^{(4)}$.

A possibilidade de continuidade do tratamento de saúde na privacidade do domicílio permite a retomada da rotina diária da pessoa dependente e familiares, e possível diminuição do estresse e melhora das condições de saúde, pois quando a hospitalização é prolongada há alterações nos hábitos de vida do dependente, afastando-o dos seus familiares, dos amigos, e de seus objetos pessoais, além do risco de infecção hospitalar.

A assistência domiciliar pode se apresentar de três formas: a preventiva, que busca evitar o adoecimento ou mesmo o agravamento de doenças já existentes, a terapêutica, quando o tratamento é oferecido até receber alta médica, e a paliativa, que proporciona tratamento para pessoas com patologias sem perspectiva de cura visando uma melhor qualidade de $v{ }^{(5)}$. Normalmente a modalidade de assistência terapêutica é a mais praticada, e configura-se como uma importante estratégia para que o cuidado no domicílio ocorra de forma confortável, segura e humana ${ }^{(6)}$.

A assistência domiciliar surge como alternativa que beneficia especialmente idosos com doenças incapacitantes e dependentes da ajuda de cuidadores por longo tempo, que se encarregue de seus cuidados básicos ${ }^{(3)}$.

Torna-se importante o estudo dos processos envolvidos na assistência domiciliar para identificação das necessidades das pessoas dependentes e seus cuidadores visando a melhoria da qualidade de vida dos mesmos.
Várias instituições adotam tal modalidade de atenção à saúde para atender as pessoas em seu domicílio, em consonância com a proposta da assistência domiciliar.

O Departamento de Atendimento Domiciliar/DAD de uma cooperativa de trabalho médico do interior do Estado de São Paulo presta atendimento em domicílio a pessoas de todas as faixas etárias e gênero, que se enquadram em normas previamente estabelecidas, encaminhadas do hospital ou do consultório pelo médico responsável pelo tratamento de saúde, com diagnósticos de Pneumonia, Doenças Neurológicas, Lesões de pele, Doença Pulmonar Obstrutiva Crônica, Doenças I nfecciosas, Doenças crônico-degenerativas, Doenças Neurológicas, para a realização de procedimentos como: curativo, antibioticoterapia, fototerapia, sondagem, fisioterapia, entre outros.

Os atendimentos realizados são prestados por equipe multiprofissional composta por coordenador médico, assistente social, enfermeira supervisora, fisioterapeutas, fonoaudióloga, nutricionista, técnicas de enfermagem, terapeuta ocupacional e equipe administrativa composta por assistente administrativo, recepcionista e motoristas.

Tais considerações motivaram a realização deste estudo, que teve por objetivos caracterizar o cliente em atendimento domiciliar e seu respectivo cuidador; conhecer as percepções dessa população quanto à qualidade do serviço prestado na assistência domiciliar e conhecer como o cliente avalia o cuidado prestado por seu cuidador.

\section{METODOLOGIA}

Trata-se de um estudo de caráter descritivo exploratório. A opção pela utilização de uma pesquisa de caráter exploratório fundamenta-se no fato de que ela tem por finalidade principal desenvolver, esclarecer e modificar conceitos e idéias, tendo em vista a formulação de problemas de forma mais precisa, ou ainda, formular algumas hipóteses para estudos posteriores ${ }^{(7-8)}$.

Estudo realizado nas residências dos clientes matriculados no DAD de uma cidade no interior do estado de São Paulo, no período de abril a agosto de 2006.

A coleta de dados foi realizada utilizando a técnica de entrevista individual com as pessoas dependentes e seus respectivos cuidadores na própria residência. A utilização da entrevista possibilita captação das informações imediata independente do tipo de informante e sobre os mais variados tópicos $^{(9)}$.

Inicialmente foi realizado um levantamento das pessoas dependentes matriculadas no DAD e seus respectivos cuidadores. Em seguida os cuidadores foram contatados por telefone e agendado um horário para a realização das entrevistas com aqueles que concordaram em participar do estudo. 
Foram consideradas participantes da pesquisa todas as pessoas que possuíam condições de responder a uma entrevista (excluindo-se crianças até doze anos), que estavam matriculados no departamento e que já tivessem sido atendidos pela equipe do DAD pelo menos 10 vezes antes da entrevista.

Para a realização das entrevistas foi utilizado um instrumento contendo questões abertas e fechadas, compreendendo duas partes: a primeira referente às questões dirigidas ao dependente (dados de identificação, itens relativos à qualidade do atendimento prestado, percepções quanto ao papel desempenhado pelo cuidador) e a segunda, questões dirigidas ao cuidador (dados de identificação, itens relativos às atividades por ele desempenhadas, percepções quanto à qualidade do atendimento prestado pela equipe do atendimento domiciliar).

Os dados oriundos das questões fechadas do instrumento foram organizados em uma planilha eletrônica, sendo apresentados em forma de figuras e percentuais. Após a coleta dos dados, procedeu-se à organização das informações por meio da préanálise, exploração do material, tratamento dos resultados e interpretação. As questões abertas foram analisadas e agrupadas em categorias de acordo com a similaridade dos relatos.
Para o desenvolvimento desta pesquisa foram observadas as recomendações contidas na Resolução 196/96 do Conselho Nacional de Saúde, e as entrevistas somente foram iniciadas após parecer favorável do Comitê de Ética em Pesquisa em Seres Humanos da Universidade Federal de São Carlos (Parecer n. 017/2006 de 08 de fevereiro de 2006).

Antes da realização da entrevista, as pessoas dependentes e seus cuidadores foram devidamente informados e esclarecidos sobre os propósitos do estudo, consentiram sua participação mediante assinatura do Termo de Consentimento Livre e Esclarecido.

\section{RESULTADOS E DISCUSSÃO}

Fizeram parte do estudo quarenta clientes matriculados no Departamento de Atendimento Domiciliar e que receberam pelo menos dez atendimentos da equipe antes do momento da entrevista.

\section{Questões dirigidas ao dependente}

A maioria das pessoas dependentes pertencia a faixa etária de 61 a 70 anos (25\%), seguida por clientes na faixa etária de 71 a 80 anos $(22,5 \%), 81$ a 90 anos (17,5\%), 91 a 100 anos (15\%), 51 a 60 anos (12,5\%), 11 a 20 anos $(5 \%)$ e 21 a 30 anos (2,5\%) (Gráfico 1).

Gráfico 1: Porcentagem de pessoas dependentes por faixa etária. São Carlos, 2006.

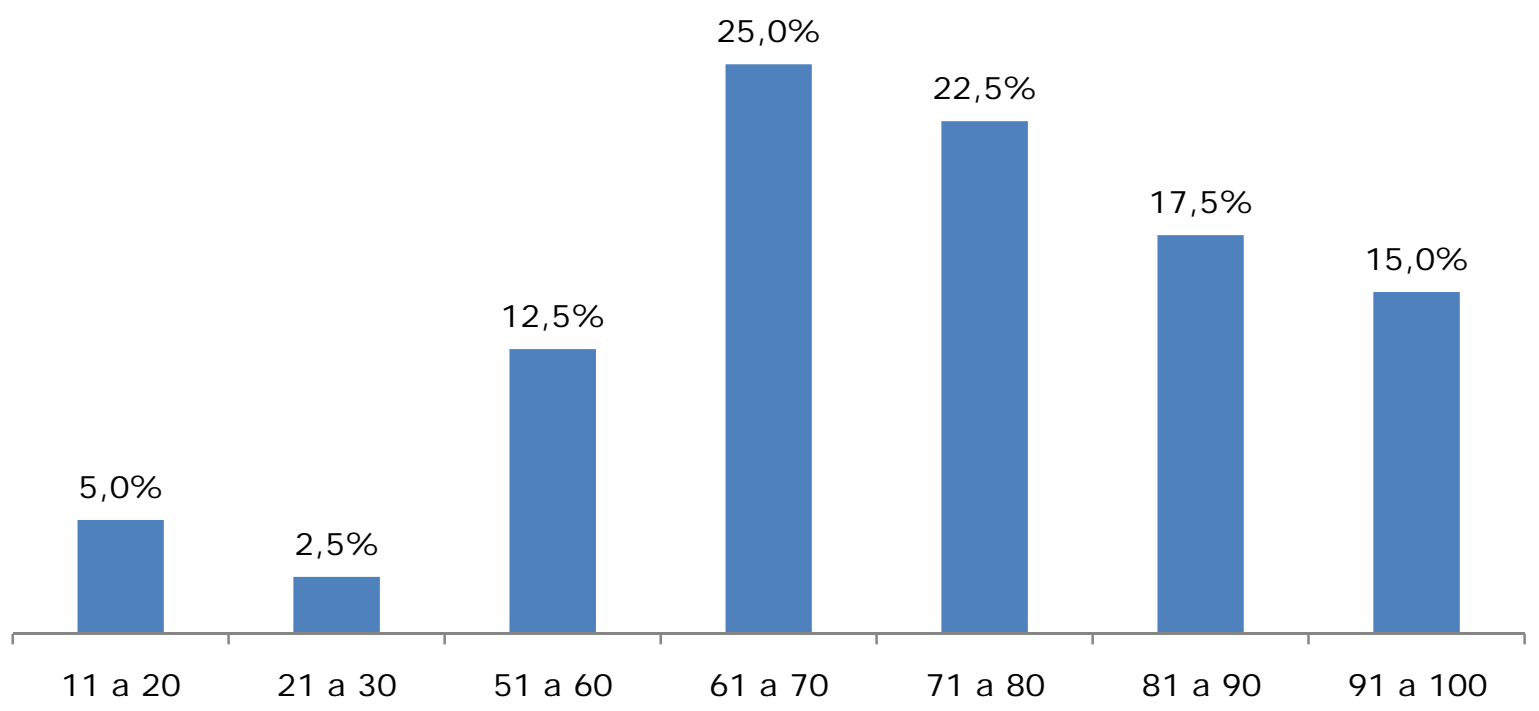

A faixa etária acima de 60 anos compunha um total de $80 \%$ dos entrevistados, fator este representativo da realidade mundial que aponta para o envelhecimento populacional com a formação de uma população idosa atingida por doenças crônicodegenerativas. Quanto ao gênero, 67,5\% das pessoas dependentes atendidas deste estudo eram mulheres.
No que se refere à profissão das pessoas dependentes, $45 \%$ eram do lar, 32,5\% eram aposentados, e $22,5 \%$ compunham a categoria outros como: cabeleireira, contador, empregada doméstica, comerciante, auxiliar de produção, estudante e professora.

Tratando-se do nível de escolaridade dos participantes, por meio do Gráfico 2 é possível observar que $35 \%$ das pessoas dependentes 
cursaram o ensino médio incompleto, 22,5\% pertenciam a categoria outro, composta por: magistério, analfabeto, curso técnico, ginásio comercial, $15 \%$ cursaram o ensino fundamental incompleto, bem como o ensino médio completo, $12,5 \%$ cursaram o ensino fundamental completo.

Gráfico 2: Porcentagem de pessoas dependentes por nível de escolaridade. São Carlos, 2006.

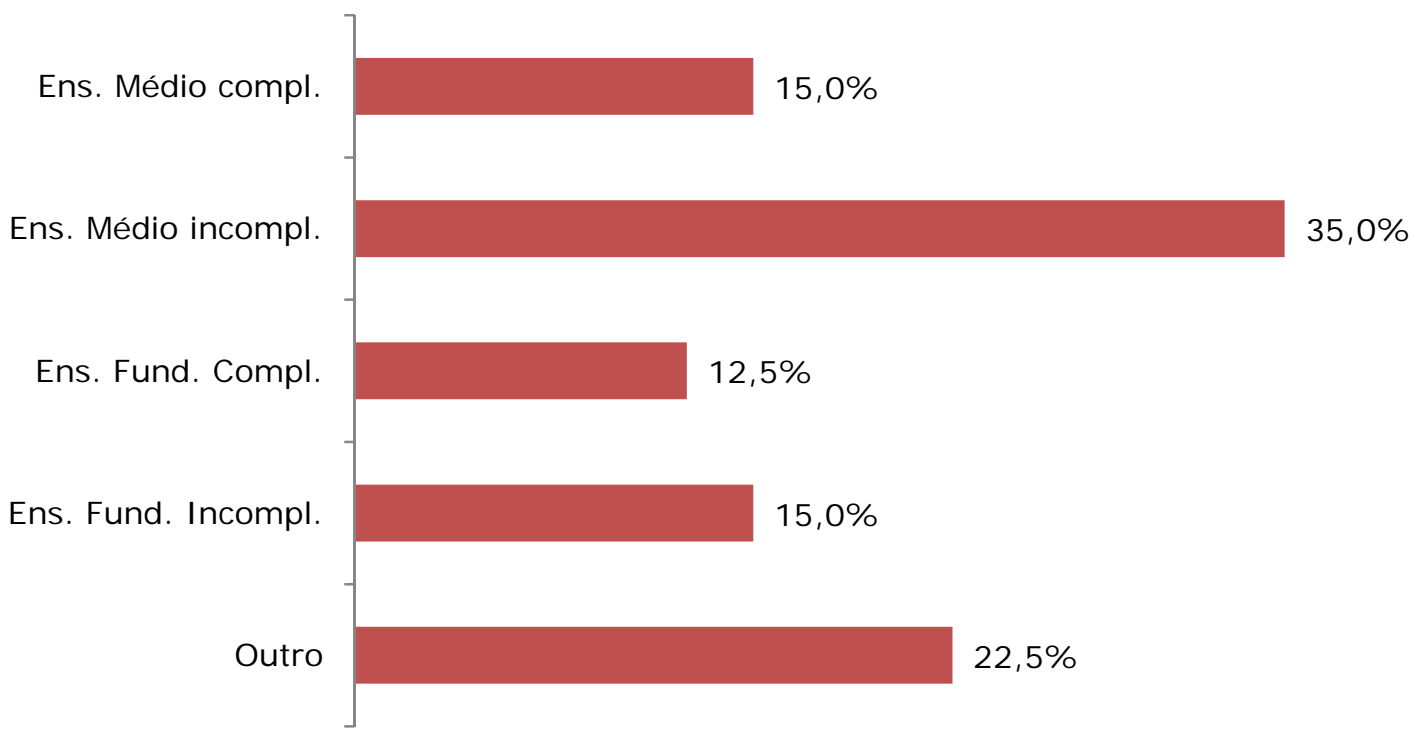

Quanto ao estado civil, 42,5\% dos clientes eram viúvos, $40 \%$ casados, $15 \%$ solteiros e $2,5 \%$ informaram outra situação.

No item patologia dos clientes, 27,5\% apresentavam Doenças Neurológicas, 22,5\%
Fraturas, $17,5 \%$ Lesões de pele, 17,5\% outras patologias como Hepatopatia, Pneumonia, Doença Pulmonar Obstrutiva Crônica, Infecção do Trato Urinário, Endocardite, Esclerose Múltipla, 7,5\% Osteomielite e 7,5\% Neoplasias (Gráfico 3).

Gráfico 3: Porcentagem de pessoas dependentes por patologia. São Carlos, 2006.

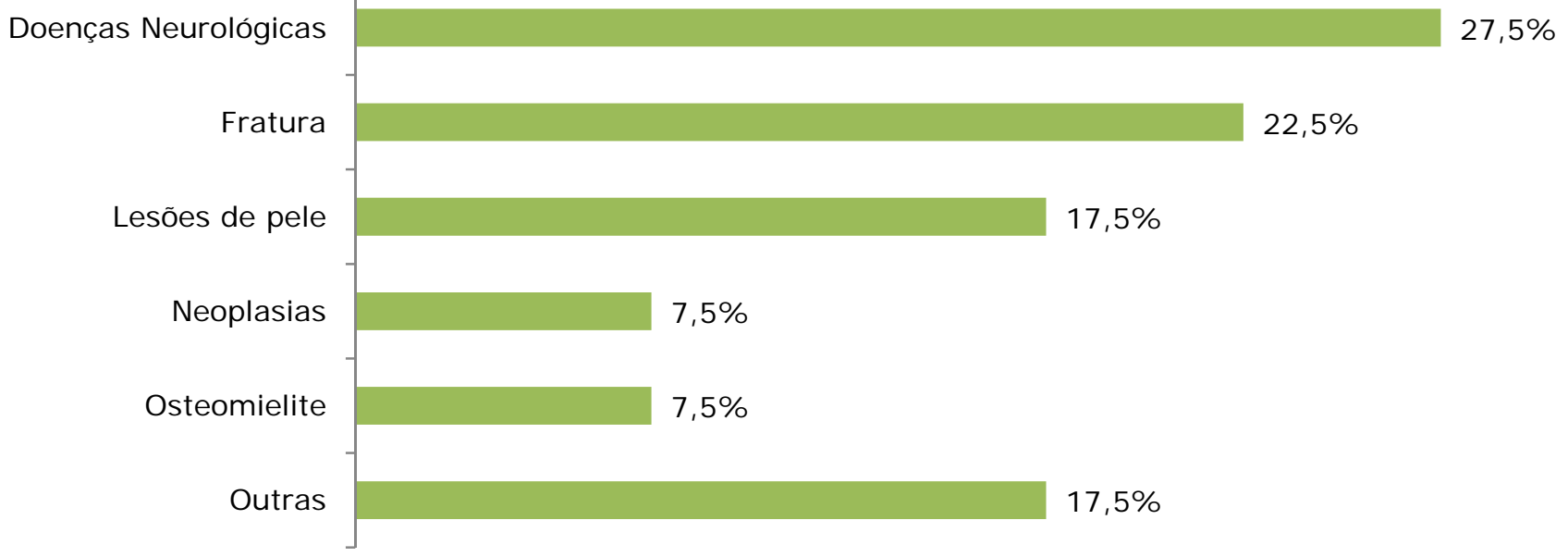

A assistência domiciliar surgiu como alternativa que beneficia especialmente idosos com doenças incapacitantes, e conseqüentemente com aumento da probabilidade de depender de cuidados de longa duração, sofrendo com a perda da independência(3).

No que tange ao tempo em que recebiam atendimento domiciliar, a maioria dos entrevistados $(62,5 \%)$ o recebiam no prazo de 0 a 6 meses, $22,5 \%$ de 6 meses a 1 ano, 2 a 3 anos, 3 a 4 anos, 6 a 7 anos, $15 \%$ de 1 a 2 anos.
As visitas domiciliares foram realizadas por diferentes profissionais, de acordo com as necessidades de cada cliente. Nesse contexto foram totalizados 72 atendimentos, realizados pelos seguintes profissionais do programa: Assistente Social: 31, Nutricionista: 29, Terapeuta Ocupacional: 24, Médicos 22, Fisioterapeutas: 22 e Fonoaudióloga: 4. No que se refere à Enfermagem, foram totalizados 77 atendimentos, realizados pela Enfermeira e pelas Técnicas de Enfermagem para a realização de 
diversos procedimentos como: curativo: 20, controle de sinais vitais: 24 , medicação: 20 , sondagem: 8 e outros: 5 .
Quanto à qualidade do atendimento prestado, a maioria dos entrevistados a consideraram ótima ou boa como se observa no Quadro 1.

Quadro 1: Opinião dos entrevistados sobre a qualidade do atendimento. São Carlos, 2006.

\begin{tabular}{|c|c|c|c|}
\hline Itens & Ótimo & Bom & Ruim \\
\hline Atendimento telefônico $*$ & 1 & 2 & - \\
\hline Educação da equipe & 33 & 7 & - \\
\hline Simpatia & 34 & 6 & - \\
\hline Clareza das orientações & 29 & 11 & - \\
\hline Atenção despendida & 28 & 9 & - \\
\hline Tranqüilidade & 31 & 9 & - \\
\hline Eficiência & 31 & 13 & - \\
\hline Assiduidade das visitas & 24 & - & - \\
\hline
\end{tabular}

* Os demais clientes relataram não ter realizado esse tipo de serviço.

As vantagens citadas foram avaliadas e agrupadas em 3 pontos convergentes a partir da fala dos sujeitos:

Atenção e cuidado da equipe

Nesta categoria foram mencionadas vantagens como a atenção prestada pelo médico e equipe no atendimento domiciliar. Foi também relatado como vantagem poder ser atendido por equipe considerada “agradável" e especializada.

A assistência domiciliar proporciona à pessoa dependente e familiares receber atendimento especializado e com privacidade, resultando na rápida recuperação da saúde ${ }^{(10)}$.

O ambiente domiciliar permite o contato mais estreito entre a equipe de saúde e o paciente e seus familiares, podendo ser esse um momento de grande utilidade para avaliar suas condições de vida, importantes para o sucesso do acompanhamento ${ }^{(11)}$.

\section{Conforto do lar}

As vantagens mencionadas nesta categoria foram relacionados à comodidade do lar, quando relatam a liberdade e tranqüilidade de estar em casa e poder permanecer com a família.

A importância da assistência domiciliar consiste em proporcionar ao ser humano a possibilidade de interagir com seu meio, de maneira equilibrada e saudável ${ }^{(5)}$.

Ter privacidade em casa, estar em um ambiente que não alimenta a idéia de enfermidade, poder contar com o apoio, atenção e carinho dos familiares são vantagens proporcionadas pela assistência domiciliar. A assistência em gerontologia preconiza a manutenção do idoso, pelo maior temopo possível, na familiaridade, conforto e dignidade de seus lares $^{(12)}$.
“Desospitalização”

Esta categoria apresenta o fato de poder sair do hospital, como uma maneira de evitar os riscos de adquirir infecção hospitalar, motivo esse apontado pela maioria dos entrevistados $(97,5 \%)$.

A pessoa dependente que continua o tratamento de saúde em casa, tem a possibilidade de retomar o mais breve possível o seu cotidiano, e ao ter controle de seu ambiente, há diminuição do estresse e melhor possibilidade de recuperação(5).

A desospitalização é associada à humanização e proporciona a reintegração do indivíduo ao contexto familiar favorecendo a sua recuperação. Em casa, a pessoa dependente sofre menor risco de adquirir infecção, evita o agravamento de doenças e reinternações ${ }^{(6)}$.

Por outro lado, quando o idoso permanece hospitalizado por muito tempo, há alterações em seus hábitos de vida, afastando-o dos seus familiares e amigos, de seus objetos pessoais, além do receio quanto a seu estado de saúde, medo da morte, dependência e novas situações passíveis de serem vividas $^{(13)}$.

Inúmeras foram as vantagens mencionadas, apenas um entrevistado mencionou o atraso do horário de atendimento de um membro da equipe como desvantagem.

Quanto às percepções dos clientes sobre 0 cuidado realizado por seus cuidadores, 87,5\% avaliaram como ótima a assistência prestada. Todos relataram que o cuidador realiza as orientações feitas pela equipe do DAD e que eles compreendem as orientações transmitidas pelo cuidador e pela equipe. A maioria $(87,5 \%)$ considerou ótima sua relação com o cuidador e os demais a consideraram boa.

\section{Questões dirigidas ao cuidador}

Participaram da pesquisa 40 cuidadores, sendo que $32,5 \%$ pertenciam a faixa etária de 51 a 60 anos, $20 \%$ de 61 a 70 anos, 17,5\% de 31 a 40 anos, 
12,5\% de 21 a 30 anos e de 41 a 50 anos, e 5\% de 71 a 80 anos (Gráfico 4).

Gráfico 4: Porcentagem de cuidadores por faixa etária. São Carlos, 2006.

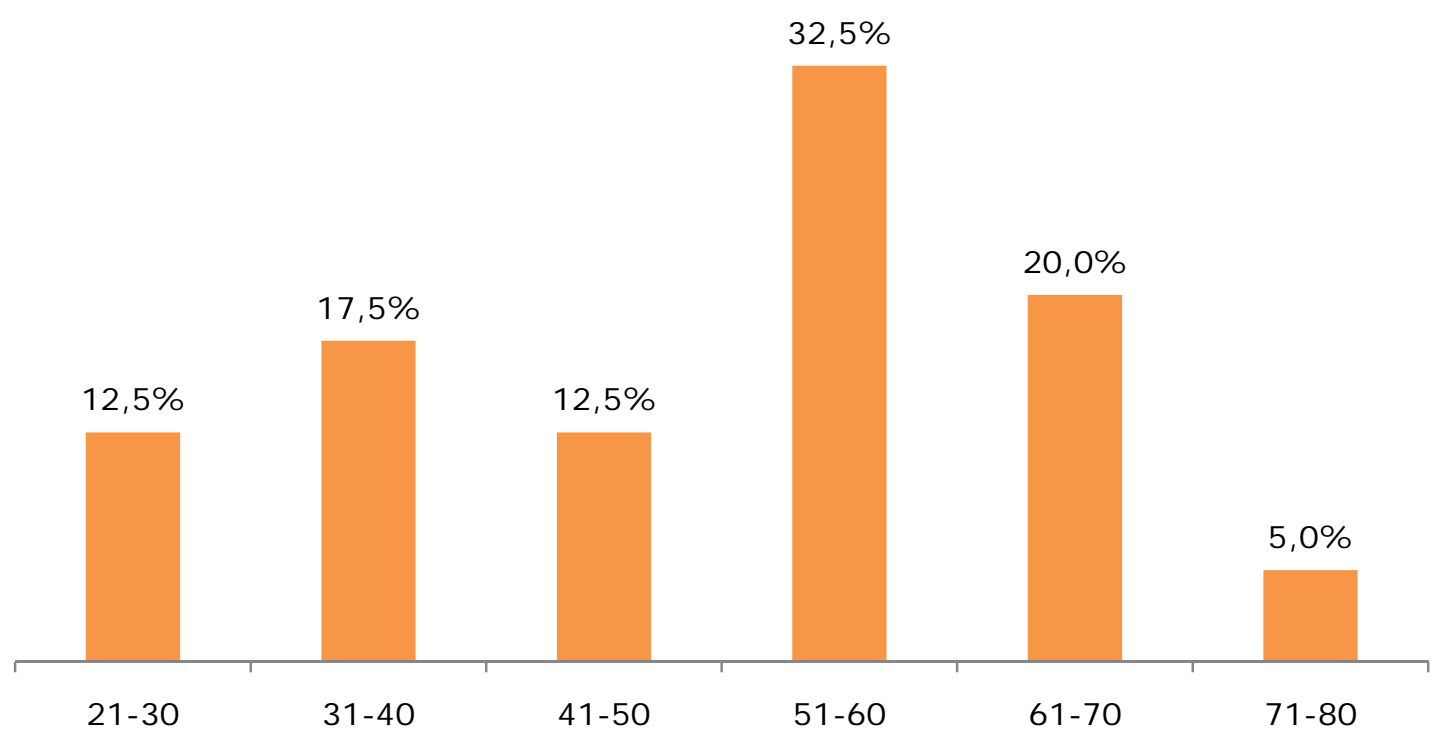

Por meio desta amostra, verificou-se que $25 \%$ dos cuidadores possuem mais que 60 anos, exercendo atividades como cuidador de idosos, função esta que requer bastante disposição física e mental acarretando significativo ônus para os mesmos.

Os cuidadores de idosos são desafiados por inúmeras demandas, previsíveis ou não, em função diminuição da capacidade funcional do idoso, aliada a presença de múltiplos fatores inerentes ao ato de cuidado, são geradoras de ônus pela família(14).

Vale ressaltar que o cuidador sente-se inseguro, temeroso e receoso em função das inúmeras atividades relacionadas ao cuidar que, geralmente, não recebeu preparo ${ }^{(12)}$.

Quanto à profissão dos cuidadores, 27,5\% eram do lar, $17,5 \%$ eram aposentados, $10 \%$ empregadas domésticas, $7,5 \%$ se auto denominaram cuidadores e 37,5\% atuavam como: artesão, bibliotecário, estudante, servidor público, professor, motorista, comerciante.

No que se refere ao nível de escolaridade, por meio do Gráfico 5, pode-se observar que a maioria dos cuidadores entrevistados não concluíram o ensino fundamental $(32,5 \%), 27,5 \%$ concluíram o ensino médio, sendo que os demais apresentaram grau de escolaridade diversos.

Gráfico 5: Porcentagem de cuidadores por nível de escolaridade. São Carlos, 2006.

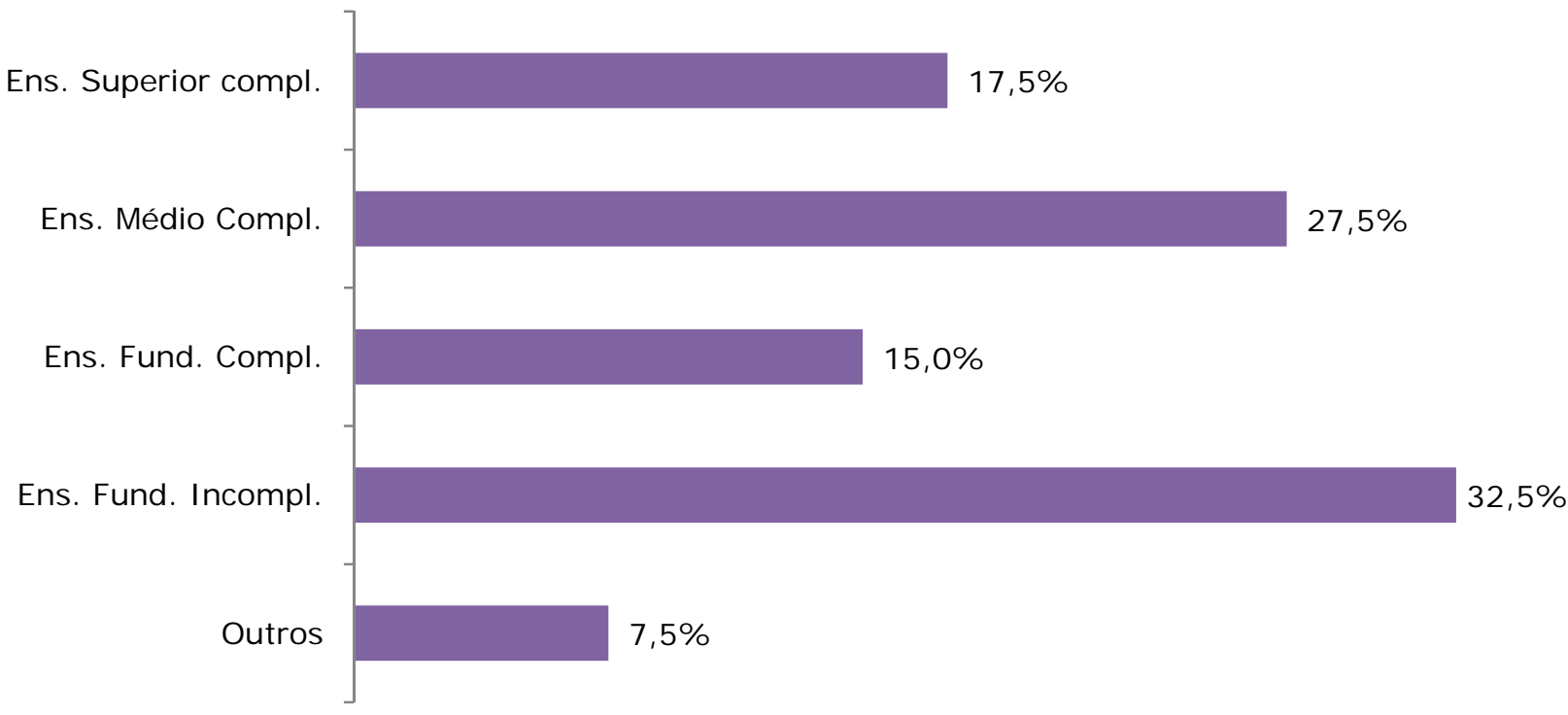


Dos cuidadores que participaram do estudo, $30 \%$ não possuíam parentesco com os dependentes, $27,5 \%$ eram cônjuges, $25 \%$ eram filhos, $10 \%$ eram genro/nora e $7,5 \%$ eram pai/mãe.

Tornar-se cuidador é um processo que ocorre gradual ou repentinamente, de acordo com a patologia do dependente e, geralmente, obedece a alguns fatores como parentesco (freqüência maior para cônjuges), gênero (predominância para mulheres), proximidade física (considerando-se quem vive com a pessoa) e proximidade afetiva (maior destaque para as relações conjugais e entre pais e filhos) ${ }^{(15-18)}$.

Em concordância com a literatura pertinente da área ${ }^{(15-16)}$, neste estudo pudemos observar a predominância do gênero feminino exercendo a função de cuidadores $(85 \%)$. Tal fato ocorre, uma vez que as mulheres normalmente assumem o cuidar por ser este um papel natural, inscrito socialmente na função de mãe e, culturalmente o assumem por ser mais um papel pertinente à esfera doméstica.

Verifica-se no Brasil que o compromisso de cuidar de uma pessoa dependente é normalmente assumido por cuidadoras, como: filha, nora, neta e sobrinha ${ }^{(17)}$.

Cuidar em família é geralmente uma tarefa desempenhada por mulheres, como a organização da vida familiar, da casa, o cuidado dos filhos e dos idosos, seguindo normas culturais que atribuem ao homem o sustento e sobrevivência material da família, assumindo o cuidado relacionado às atividades instrumentais de vida diária, especialmente as relacionadas à administração de finanças ${ }^{(17-18)}$.

Os dados revelaram que $62,5 \%$ dos cuidadores eram casados, $17,5 \%$ solteiros, $12,5 \%$ divorciados e $7,5 \%$ viúvos.

Quanto ao tempo em que exerciam a função de cuidadores, 35\% o realizavam de 0 a 6 meses, $17,5 \%$, de 1 a 2 anos, $10 \%$, de 4 a 5 anos, 37,5\% outro como: 6 meses a 1 ano, 3 a 4 anos, 5 a 6 anos, 6 a 7 anos, 8 a 9 anos, 9 a 10 anos e acima de 10 anos.

É importante ressaltar que os cuidadores relataram exercer sua função por tempo superior ao mencionado pelos clientes, provavelmente porque tratando-se de idosos, o apoio nas atividades cotidianas ocorre de maneira progressiva, considerando-se doenças crônico-degenerativas, que envolvem exigências crescentes de acordo com as necessidades dos mesmos.

Com o avanço da idade as pessoas podem debilitar-se gradativamente devido às alterações fisiológicas, limitando as funções do organismo. Isto faz com que se tornem predispostos à depender de outra pessoa para realizar o autocuidado, com conseqüente perda de autonomia e de qualidade de

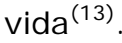

Quanto ao número de horas dedicadas à pessoa dependente, a maioria dos cuidadores $(77,5 \%)$ o fazia em tempo integral. Outros $22,5 \%$ dedicavamse de 8 a 12 horas, $5 \%$ por $8,10,12,16$ horas cada e 2,5\% por 14 horas. Ressalta-se que a maioria dos cuidadores $(77,5 \%)$ residia com a pessoa dependente.

Com relação às atividades desempenhadas pelos cuidadores, cada cuidador relatou realizar mais do que uma, sendo que a maioria deles (35), mencionou o preparo da alimentação como a principal atividade.

Cabe ao cuidador a execução de atividades básicas de vida diária que envolvem ações como alimentar, vestir, banhar, mobilizar, deambular a pessoa dependente entre outras, bem como as atividades instrumentais de vida diária, ou seja, aquelas relacionadas à capacidade da pessoa levar uma vida independente na comunidade (realizar compras, administrar medicações, administrar finanças, utilizar transporte, entre outras) (Quadro 2).

Quadro 2: Atividades básicas e instrumentais de vida diária, desempenhadas pelo cuidador. São Carlos, 2006.

\begin{tabular}{|c|c|}
\hline Atividades & No. de cuidadores que a desempenham \\
\hline Preparo de alimentação & 35 \\
\hline Marcação de consultas & 30 \\
\hline Banho & 29 \\
\hline Administração de medicação & 29 \\
\hline Locomoção & 27 \\
\hline Vestuário & 27 \\
\hline Higiene pessoal & 26 \\
\hline Compra de medicação & 25 \\
\hline Limpeza da casa & 24 \\
\hline Pagamentos & 23 \\
\hline Administração das finanças & 18 \\
\hline Mudança de decúbito & 17 \\
\hline Exercícios & 15 \\
\hline Oferecer alimentação & 13 \\
\hline Outras & 4 \\
\hline
\end{tabular}


Apoio psicológico e companhia foram atividades citadas por dois cuidadores, embora ocorram naturalmente na vida da maioria dos participantes.

As atividades realizadas pelo cuidador à pessoa dependente, ocorrem de acordo com o grau de incapacidade e não necessariamente de acordo a sua patologia.

As atividades básicas de vida diária como banhar, auxiliar a locomoção e vestir a pessoa dependente são, geralmente, mais difíceis de serem realizadas por idosos em função das limitações físicas e biológicas inerentes a faixa etária.

Quanto aos itens relativos à qualidade do atendimento prestado a maioria dos cuidadores relatou satisfação com o atendimento da equipe do DAD (Quadro 3).

Quadro 3: I tens relativos a qualidade do atendimento. São Carlos, 2006.

\begin{tabular}{|c|c|c|c|}
\hline I tens & Ótimo & Bom & Ruim \\
\hline Atendimento telefônico* & 11 & 15 & - \\
\hline Educação da equipe & 31 & 8 & 1 \\
\hline Simpatia & 34 & 6 & - \\
\hline Clareza das orientações & 29 & 11 & - \\
\hline Atenção dispendida & 30 & 10 & - \\
\hline Tranqüilidade & 30 & 10 & - \\
\hline Eficiência & 29 & 11 & - \\
\hline Assiduidade das visitas & 26 & 10 & 4 \\
\hline Atendimento no DAD* & 13 & 3 & - \\
\hline
\end{tabular}

* Os demais cuidadores relataram não ter realizado esse tipo de serviço.

As vantagens do atendimento domiciliar citadas pelos participantes da pesquisa foram analisadas e agrupadas em três pontos convergentes a partir da fala dos participantes:

Atenção e cuidado da equipe

Nesta categoria foram mencionadas como vantagens do atendimento domiciliar aspectos relacionados à postura da equipe, como o carinho, a atenção e o respeito dispensados aos pacientes e seus cuidadores. O serviço de atendimento domiciliar também foi considerado atencioso.

Relataram que o atendimento foi personalizado e, por receberem a equipe em casa, sentiam-se tratados como amigos.

A equipe foi considerada capacitada, especializada e eficiente, fatores esses geradores de sentimentos de segurança e confiabilidade.

Em várias situações foram fornecidas orientações aos familiares por telefone, o que foi citado pelos cuidadores como fator importante para a resolução de alguns questionamentos. Mencionaram também a satisfação em sentir a equipe pronta para atender suas necessidades.

\section{Conforto do lar}

Nesta categoria foi relatada como vantagem, pela maioria dos cuidadores, o fato de haver atendimento no próprio domicílio, sem a necessidade de locomover a pessoa dependente até um serviço de saúde. Isso, segundo os cuidadores, gera comodidade e conforto tanto para eles como para a pessoa dependente.

A permanência da pessoa com dependência em casa com a família é fundamental segundo os cuidadores, pois permite a manutenção da rotina da casa, o que não seria possível em caso de internação da mesma. Alguns cuidadores mencionaram as vantagens psicológicas para o cliente e cuidador provenientes da assistência no próprio domicílio.

Permanecer no próprio domicílio, no "conforto do lar", foi mencionado pelos cuidadores como uma vantagem, gerando sentimentos de segurança e tranqüilidade, sem custos adicionais.

\section{Desospitalização}

As vantagens mencionadas pelos cuidadores referiram-se à possibilidade de realizar o tratamento no próprio domicílio, pois quando comparado ao hospital, essa condição previne doenças e diminui riscos de contaminação.

Ao mencionarem os benefícios da "desospitalização", os cuidadores deram maior ênfase aos riscos inerentes ao ambiente hospitalar enquanto que os clientes ressaltaram o desejo de retornar ao ambiente familiar.

Para o cuidador, cuidar da pessoa dependente em casa elimina o deslocamento do mesmo até o hospital, além de deixar de "gastar dinheiro" e tempo com locomoção.

Além das vantagens já conhecidas da assistência domiciliar como maior humanização no tratamento, diminuição de internações e do tempo de permanência em hospitais, deve-se considerar um benefício muito grande, porém de difícil mensuração que seria a satisfação da pessoa dependente e familiares ao realizar seu tratamento de saúde no próprio domicílio ${ }^{(19)}$.

A maioria dos cuidadores (90\%) não mencionou desvantagens quanto ao atendimento domiciliar. O 
fato do DAD não prestar atendimento de urgência e emergência no domicílio foi mencionado como desvantagem por uma cuidadora .

Como o propósito do atendimento domiciliar é realizar ações programadas e previamente agendadas, os clientes cadastrados são orientados a procurar um serviço especializado quando necessitarem de um atendimento de urgência.

Uma cuidadora mencionou como desvantagem a falta de liberdade que sentia, pois sua sogra recebia visita diária dos profissionais do DAD para realização de curativo, e ela tinha que permanecer em casa até a chegada da equipe. Normalmente as visitas para realização de curativos são agendadas com as famílias sem um horário previamente estabelecido, sendo comunicadas sobre o período (manhã ou tarde). I sso não acontece com as visitas para administração de medicamentos, pois os mesmos são prescritos pelo médico, devendo-se respeitar os horários para a eficácia do tratamento.

Foi relatado descontentamento por parte de uma cuidadora quanto ao horário de aplicação de medicação quando este ocorria de madrugada, seguindo prescrição médica e intervalo de medicação.

Uma cuidadora mencionou a falta de autonomia da família em solicitar consulta médica domiciliar sem consentimento do médico responsável pelo tratamento de saúde, que consiste em norma do Departamento de Atendimento Domiciliar. A família tem liberdade para agendar consulta médica domiciliar desde que se responsabilize pelos custos gerados pela visita, fato este esclarecido no momento de inclusão do dependente no DAD.

\section{CONSI DERAÇÕES FI NAIS}

A assistência domiciliar consiste na oferta de serviços que visam a individualização da assistência prestada pelas instituições de saúde, bem como a autonomia da pessoa dependente e seus familiares quanto ao planejamento e execução dos cuidados necessários.

Quando a hospitalização é prolongada, há alterações nos hábitos de vida da pessoa dependente, afastando-o do convívio dos seus familiares, amigos, e de seus objetos pessoais, além do risco de infecção hospitalar. Assim sendo, receber assistência à saúde no próprio domicílio permite a retomada da rotina diária da pessoa dependente e seus respectivos familiares, em um ambiente que não alimenta a idéia de doença e ainda permite a conciliação dos cuidados com as demais atividades domésticas, familiares e/ou profissionais.

A privacidade do domicílio, como também o apoio, a atenção e o carinho dos familiares são vantagens proporcionadas pela assistência domiciliar, favorecendo a recuperação do estado de saúde da pessoa dependente.
Assim como em diversos estudos da área, observa-se que as atividades realizadas no cuidado da pessoa dependente são desempenhadas, na maioria das vezes, por cuidadores do gênero feminino, sendo esta uma função atribuída cultural e socialmente.

A satisfação gerada pela assistência domiciliar consiste em vantagem não mensurável, porém, constantemente observada pela equipe do DAD e comprovada por meio do relato dos participantes deste estudo nos diferentes aspectos pesquisados.

A assistência prestada pelo cuidador foi considerada satisfatória na visão da pessoa dependente, uma vez que os mesmos seguem as prescrições médicas e orientações da equipe do DAD.

Avaliar a qualidade do atendimento prestado permite a reflexão de pontos a serem revisados bem como a adequação dos atendimentos frente às necessidades da clientela e seus cuidadores. Esta tem sido uma preocupação constante da equipe do DAD com vista a proporcionar a melhora contínua da qualidade do serviço, e conseqüente satisfação dos clientes e seus cuidadores.

\section{REFERÊNCI AS}

1. Albuquerque SMRL. Qualidade de vida do idoso: a assistência domiciliar faz a diferença? São Paulo: Casa do Psicólogo; 2003.

2. Ministério da Saúde; Agência Nacional de Vigilância Sanitária (ANVISA). Resolução Diretoria Colegiada n. 11, de 26 de janeiro de 2006. Dispõe sobre o regulamento técnico para o funcionamento de serviços que prestam atenção domiciliar. Brasília (Brasil): Ministério da Saúde; 2006.

3. Rodrigues MR, Almeida RT. Papel do responsável pelos cuidados à saúde do dependente no domicílio: um estudo de caso. Acta paul. enferm. 2005; 18(1): 20-4.

4. Duarte YAO, Diogo MJD'E. Atendimento domiciliário: um enfoque gerontológico. In: Duarte YAO, Diogo MJD’E (Org.). Atendimento domiciliário: um enfoque gerontológico. São Paulo: Editora Atheneu; 2000. p.3-17.

5. Ceschini M. Porque assistência domiciliar. In: Dias ELF (Org.). Orientações para cuidadores informais na assistência domiciliar. Campinas: Editora da Unicamp; 2002. p. 11-16.

6. Sena RR et al. O cuidado no domicílio: um desafio em construção. Cogitare Enferm. 1999; 4(2): 58-62.

7. Seltiz $C$ et al. Métodos de pesquisa nas relações sociais. São Paulo: EPU-Edusp; 1967.

8. Gil AC. Métodos e técnicas da pesquisa social. São Paulo: Atlas; 1995.

9. Lüdke M, André MED. A pesquisa em educação: abordagens qualitativas. São Paulo: EPCL; 1988.

10. Carvalho TA, Siefer MW. Pesquisa de opinião de serviços de assistência domiciliar contratados no pósalta hospitalar ("home care"). Revista da Sociedade 
de Cardiologia do Estado de São Paulo. 2004; 14(5): 10-18.

11. Paskulin LMG, Dias VRFG. Como é ser cuidado em casa: as percepções dos clientes. Rev Bras Enferm. 2002; 55(2): 140-5.

12. Diogo MJ'E, Duarte YAO Cuidados em domicílio: conceitos e práticas. In: Freitas EV et al. Tratado de geriatria e gerontologia. 2nd ed. Rio de Janeiro: Guanabara Koogan; 2006. p.1122-30.

13. Marin MJS, Caetano FB, Palasson RR. Avaliação da satisfação dos usuários do Programa Interdisciplinar de Internação Domiciliar. Espaço para Saúde [Internet]. 2001 [cited 2009 feb 16];2(2). Available from: http://www.ccs.uel.br/espacoparasaude/v2n2/doc/do miciliar.htm.

14. Perracini MR, Neri AL. Tarefas de cuidar: com a palavra, mulheres cuidadoras de idosos de alta dependência. In: Neri NA (Org.). Cuidar de idosos no contexto da família. Questões psicológicas e sociais. Campinas: Editora Alínea; 2002. p. 135-163.

15. Mendes PBMT. Cuidadores: heróis anônimos do cotidiano, em São Paulo/SP [dissertation]. São Paulo: Pontifícia Universidade Católica de São Paulo; 1995. $195 \mathrm{p}$.

16. Mendes PBMT. Quem é o cuidador? In: Dias ELF (Org.). Orientações para cuidadores informais na assistência domiciliar. Campinas: Editora da Unicamp; 2002. p.19-33.

17. Pavarini $\mathrm{SCl}$ et al. Quem irá empurrar minha cadeira de rodas? A escolha do cuidador familiar do idoso. Rev. Eletr. Enf. [Internet]. 2006 [cited 2009 feb 16];8(3):326-335. Available from: http: http://www. fen.ufg. br/revista/revista8_3/v8n3a03.htm.

18. Neri NA, Sommerhalder C. As várias faces do cuidado e do bem-estar do cuidador. In: Neri NA (Org.). Cuidar de idosos no contexto da família. Questões psicológicas e sociais. Campinas: Editora Alínea; 2002. p. 9-63.

19. Fabrício SCC et al. Assistência domiciliar: a experiência de um hospital privado no interior paulista. Rev Latino-am Enfermagem. 2004; 12(5): 721-6.

Artigo recebido em 05.09.07.

Aprovado para publicação em 31.03.09. 